\title{
Estado nutricional de crianças residentes em comunidades rurais: uma revisão integrativa de literatura
}

\author{
Nutritional status of children resident in rural communities: an integrative literature review \\ Estado nutricional de los niños residentes en comunidades rurales: una revisión integrativa de la
}

Thaís da Conceição Pereira

ORCID: https://orcid.org/0000-0002-1022-7466 Universidade Regional do Cariri, Brasil

E-mail: thaispereira1914@gmail.com

Yasmin Ventura Andrade Carneiro

ORCID: https://orcid.org/0000-0002-1340-1650

Universidade Regional do Cariri, Brasil

E-mail: yasmin.ventura@urca.br

Laurineide Rocha Lima

ORCID: https://orcid.org/0000-0003-0908-6210

Universidade Regional do Cariri, Brasil

E-mail: laurineidelima@gmail.com

Edilma Gomes Rocha Cavalcante

ORCID: https://orcid.org/0000-0002-6861-2383

Universidade Regional do Cariri, Brasil E-mail: edilma.gomes@urca.br

Sandra Mara Pimentel Duavy

ORCID: https://orcid.org/0000-0002-1786-2294

Universidade Regional do Cariri, Brasil E-mail: sandra.duavy@urca.br

Simone Soares Damasceno

ORCID: https://orcid.org/0000-0002-2841-7815

Universidade Regional do Cariri, Brasil

E-mail: simone.damasceno@urca.br

Izabel Cristina Santiago Lemos de Beltrão

ORCID: https://orcid.org/0000-0002-3236-5616

Universidade Regional do Cariri, Brasil

E-mail: izabel.lemos@urca.br

\begin{abstract}
Resumo
A desnutrição ainda é um desafio para diversas nações, perdurando entre crianças oriundas de comunidades socialmente mais vulneráveis, tais como as comunidades rurais. Tem por objetivo descrever o estado nutricional de crianças residentes em comunidades rurais. Trata-se de uma revisão integrativa de literatura, realizada nos meses de setembro a dezembro de 2020, nas bases de dados Web of Science (WOS). As informações coletadas foram organizadas e compiladas eletronicamente em planilha do programa Microsoft Office Excel@versão2013, sendo validadas por dupla digitação, visando conferir maior confiabilidade. O estado nutricional de desnutrição foi observado na maioria dos estudos, indicando que as crianças em comunidades rurais, com pais não escolarizados, em condições inadequadas de moradia, vivendo em zonas de conflito e vulnerabilidade socioeconômica encontram-se mais susceptíveis à insegurança alimentar infantil. Nesse sentido, salienta-se a relevância de políticas públicas bem estruturadas e sistematicamente efetivas de inclusão social, no combate à pobreza e com foco para a redução da insegurança alimentar, visando promover a disponibilidade, o acesso ${ }^{\circ} \mathrm{ZXX}$ 'e o consumo de alimentos ricos em nutrientes.
\end{abstract}

Palavras-chave: Nutrição; Crianças; Comunidade rural; Atenção Primária à Saúde.

\section{Abstract}

Malnutrition is still a challenge for many nations, persisting among children from more socially vulnerable communities, such as rural communities. It aims to describe the nutritional status of children living in rural communities. This is an integrative literature review, in which the search for articles and sample selection will take place between September and December 2020, in which the Web of Science (WOS) databases were used. The information collected was electronically organized and compiled into a spreadsheet of the Microsoft Office Excel@version2013 program, being validated by double typing, in order to provide greater reliability. The nutritional 
status of malnutrition was observed in most studies, indicating that children in rural communities, with uneducated parents, in inadequate housing conditions, living in conflict zones, and socioeconomic vulnerability, are more susceptible to child food insecurity. In this sense, the relevance of well-structured and systematically effective public policies for social inclusion is highlighted, in the fight against poverty and with a focus on reducing food insecurity, aiming to promote the availability, access, and consumption of foods rich in nutrients.

Keywords: Nutrition; Children; Rural community; Primary Health Care.

\section{Resumen}

La desnutrición sigue siendo un desafío para muchas naciones, y persiste entre los niños de comunidades más vulnerables socialmente, como las comunidades rurales. Tiene como objetivo describir el estado nutricional de los niños que viven en comunidades rurales. Se trata de una revisión integradora de la literatura, en la que la búsqueda de artículos y selección de muestras se realizará entre septiembre y diciembre de 2020, en la que se utilizaron las bases de datos Web of Science (WOS). La información recolectada fue organizada electrónicamente y compilada en una hoja de cálculo del programa Microsoft Office Excel® versión 2013, siendo validada mediante doble mecanografía, con el fin de brindar mayor confiabilidad. El estado nutricional de desnutrición se observó en la mayoría de los estudios, lo que indica que los niños de comunidades rurales, con padres sin educación, en condiciones de vivienda inadecuadas, que viven en zonas de conflicto y vulnerabilidad socioeconómica, son más susceptibles a la inseguridad alimentaria infantil. En este sentido, se destaca la relevancia de políticas públicas bien estructuradas y sistemáticamente efectivas para la inclusión social, en la lucha contra la pobreza y con enfoque en la reducción de la inseguridad alimentaria, con el objetivo de promover la disponibilidad, acceso y consumo de alimentos ricos en nutrientes.

Palabras clave: Nutrición; Niños; Comunidad rural; Primeros auxilios.

\section{Introdução}

As experiências nos primeiros anos de vida da criança são primordiais para a formação e desenvolvimento da fase adulta. A participação entre os pais, a comunidade e os profissionais de saúde, de assistência social e de educação são imprescindíveis para a promoção da saúde, educação e desenvolvimento integral da criança. É também durante essa fase que o ser humano responde melhor aos estímulos que adquire do meio ambiente e às intervenções, quando fundamentais (Brasil, 2020).

De acordo com a Organização Mundial da Saúde (OMS), entre os anos de 1990-2016, a taxa mundial de mortalidade de crianças menores de 5 anos reduziu mais da metade (56\%), diminuindo de 93 a 41 mortes por cada 1.000 nascidos vivos. Porém, segundo esta mesma organização, apesar do progresso global na redução da mortalidade, muitas dessas mortes ocorreram em virtude de doenças que poderiam ter sido evitadas ou tratadas se houvesse acesso a intervenções simples e acessíveis (OMS, 2019).

Ainda no que diz respeito à taxa de mortalidade, existem disparidades entre regiões e países. No ano de 2018, o risco de uma criança morrer antes de completar cinco anos de idade foi maior no continente africano (76 por 1000 nascidos vivos), cerca de 8 vezes mais elevado em comparação ao continente europeu (9 por 1000 nascidos vivos) (WHO, 2018).

Nesse sentido, trazendo atenção à desnutrição - embora esteja ocorrendo a diminuição do número de crianças desnutridas -, apenas na África existem 59 milhões de crianças com até cinco anos de idade que apresentam baixa estatura para a idade. Isso representa 30,3\% das crianças nessa faixa etária. Ainda nesse segmento demográfico, 13,8 milhões de crianças têm baixo peso para a altura. A análise também mostrou que 38,0\% de todas as mulheres africanas em idade reprodutiva 110 milhões de mulheres - estavam com anemia, importante ressaltar que a desnutrição entre elas pode ter impacto na saúde dos filhos, quando decidem engravidar. Entre as crianças com até seis meses de idade, somente 43,5\% foram alimentadas exclusivamente com leite materno (FAO, 2018).

$\mathrm{Na}$ Inglaterra, a taxa de sobrepeso e a obesidade infantil são duas vezes mais elevadas em famílias localizadas em áreas mais pobres. Essas áreas mais pobres possuem cinco vezes mais restaurantes de fast food do que as mais ricas. Em diversos casos, alimentos saudáveis podem ter um custo mais elevado do que os não saudáveis, o que dificulta o acesso à alimentação saudável para as famílias de baixa renda (UNICEF, 2019). No tocante à epidemiologia da obesidade infantil, 
estima-se que em 2030 serão 234 milhões de crianças e adolescentes entre 5 e 14 anos, com obesidade no mundo (BRASIL, 2019).

Neste campo de investigação, vale mencionar o conceito de Segurança Alimentar e Nutricional (SAN) definido como a garantia de acesso contínuo de quantidade e qualidade suficientes de alimentos, obtido por meio socialmente aceitável, de forma a assegurar o bem-estar e a saúde dos indivíduos (CONSEA, 2004).

Por outro lado, a Insegurança Alimentar (IA) relaciona-se com a vulnerabilidade social por resultar de uma combinação de fatores que interferem no bem-estar das famílias e comunidades (Vieira et al., 2015). A IA está fortemente relacionada à pobreza e às desigualdades sociais. Indivíduos que residem em domicílios em insegurança alimentar, comumente, consomem uma dieta monótona e pobre em nutrientes (Vasconcelos et al., 2015).

A redução da prevalência da desnutrição infantil, em diversas regiões do mundo, inclusive no Brasil, está associada com melhores condições sociais, educativas e econômicas. Essas sensíveis mudanças observadas nos indicadores de saúde associam-se à expansão da cobertura da atenção básica, ao acesso à vacinação, ao aumento nas taxas de aleitamento materno, ao nível de escolaridade da mãe e à redução da pobreza obtida pelo programa Bolsa Família (Brasil, 2015).

No que se refere à avaliação e ao monitoramento da situação alimentar e nutricional da população brasileira, é importante destacar as ações de vigilância alimentar e nutricional realizadas com os usuários do SUS. Tais ações fazem parte das rotinas de atendimentos na rede básica de saúde, podendo citar o Sistema de Vigilância Alimentar e Nutricional (SISVAN), que tem como objetivo a detecção precoce de situações de risco nutricional permitindo definir ações que possibilitem a prevenção de agravos à saúde e retornar ao quadro de normalidade quando possível (Brasil, 2008).

Essas ações se somam com outras políticas públicas para melhorar a qualidade de vida das crianças, como no caso da criação da Política Nacional de Atenção Integral à Saúde da Criança (PNAISC) que tem como finalidade promover e proteger a saúde da criança e o aleitamento materno, mediante atenção e cuidados integrais e integrados da gestação aos 9 (nove) anos de vida, com enfoque à primeira infância, visando à redução da morbimortalidade e um ambiente facilitador à vida (Brasil, 2018).

Salienta-se, portanto, que o acompanhamento do crescimento e do desenvolvimento infantil é realizado por meio dos índices antropométricos, que permitem a classificação do estado nutricional, visto que o desequilíbrio entre as necessidades energéticas e a ingestão de alimentos provoca alterações físicas nos indivíduos, desde quadros de desnutrição e obesidade, configurando-se como informações cruciais no monitoramento das condições de saúde e de nutrição da criança (Brasil, 2011).

Todavia, a desnutrição ainda é um desafio para diversas nações, sofrendo disparidades de concentração que oscilam entre diferentes cidades, nos mais diversos países, perdurando, principalmente, entre crianças oriundas de comunidades socialmente mais vulneráveis (Vieira et al., 2010; Silveira et al., 2012). Em algumas comunidades rurais, é comum a falta de alimentos, seja por renda insuficiente para aquisição de itens básicos ou por não possuírem bens de produção necessários: terra, insumos, equipamentos, etc. (Nobre et al., 2015).

Nesse sentido, alguns estudos vêm mostrando que a população rural, retrata alta prevalência de insegurança alimentar e nutricional, determinada pela baixa renda familiar, alimentação monótona e insuficiente, essas dificuldades, contudo, vêm atreladas a outros desafios, tais como: obtenção de serviços de educação, saneamento básico, saúde e assistência técnica no campo (Morais et al., 2014; Almeida et al., 2017).

Assim, é considerando a vulnerabilidade dessa população que surge este trabalho, não no sentido técnico da construção de valores de referência, mas na diligência de estruturação e sistematização da informação disponível na literatura com dados abrangentes sobre a situação nutricional de crianças residentes em comunidades rurais.

Assim tem-se por objetivo descrever o estado nutricional de crianças residentes em comunidades rurais a partir de um recorte temporal e geográfico. 


\section{Metodologia}

Trata-se de revisão integrativa, cujo método é utilizado para sintetizar resultados alcançados com pesquisas acerca de determinado tema, de forma ampla e ordenada. O revisor/pesquisador consegue preparar uma revisão integrativa com finalidades diferentes, direcionando para a definição de conceitos, revisão de teorias ou análise metodológica (Ercole et al., 2014).

Para a estruturação da revisão integrativa é necessário seguir seis etapas para a sua elaboração, sendo elas identificação do tema e seleção da hipótese ou questão de pesquisa; estabelecimento de critérios para inclusão e exclusão de estudos/ amostragem ou busca na literatura; definição das informações a serem extraídas dos estudos selecionados/ categorização dos estudos; interpretação dos resultados; e apresentação da revisão/síntese do conhecimento (Mendes et al., 2008).

Na primeira etapa, formulou-se a seguinte questão norteadora: Qual o estado nutricional das crianças $(\leq 10$ anos) residentes em comunidades rurais?

A operacionalização da pesquisa iniciou-se com a definição dos critérios de inclusão, têm-se artigos publicados em texto completo e com acesso aberto, nos idiomas inglês, espanhol, francês e português, estabelecendo um recorte temporal para inclusão publicados no período de janeiro de 2015 a setembro de 2020.

Foram critérios de exclusão: artigos duplicados - considerando as bases consultadas, estudos com resultados e análises inconclusivas (projetos de pesquisa, projetos de pesquisa com resultados parciais), estudos do tipo revisão de literatura e os qualitativos.

Para a construção do conhecimento acerca da temática foram utilizadas as bases de dados Web of Science (WOS). Na Web of Science foi aplicado o formulário de pesquisa avançada, com associação intercalada de termos: "Nutrition”, "Children" e "Rural Population". A associação ocorreu da seguinte forma, mediante a seleção por tópicos (TS): TS= (Nutrition AND Children AND Rural Population).

Inicialmente, foram identificadas 289 referências primárias na base de dados selecionada (Figura 1).

Após a identificação, 220 estudos foram excluídos pelo processo de triagem, por meio da análise dos títulos e resumos segundo os critérios de inclusão e exclusão. Em seguida, dos 69 artigos selecionados elegíveis para a leitura na íntegra.

Para relatar o processo de identificação, triagem, elegibilidade e inclusão de estudos utilizou-se o instrumento Preferred Reporting Items for Systematic Review and Meta-Analyses (PRISMA), conforme representado na Figura 1. 
Figura 1 - Fluxograma PRISMA de artigos encontrados na Revisão Integrativa de Literatura com os descritores/palavra-chave: "Nutrition", "Children", "Rural Population". Na base de dados Web of Science (WOS). Crato, Ceará, 2020.

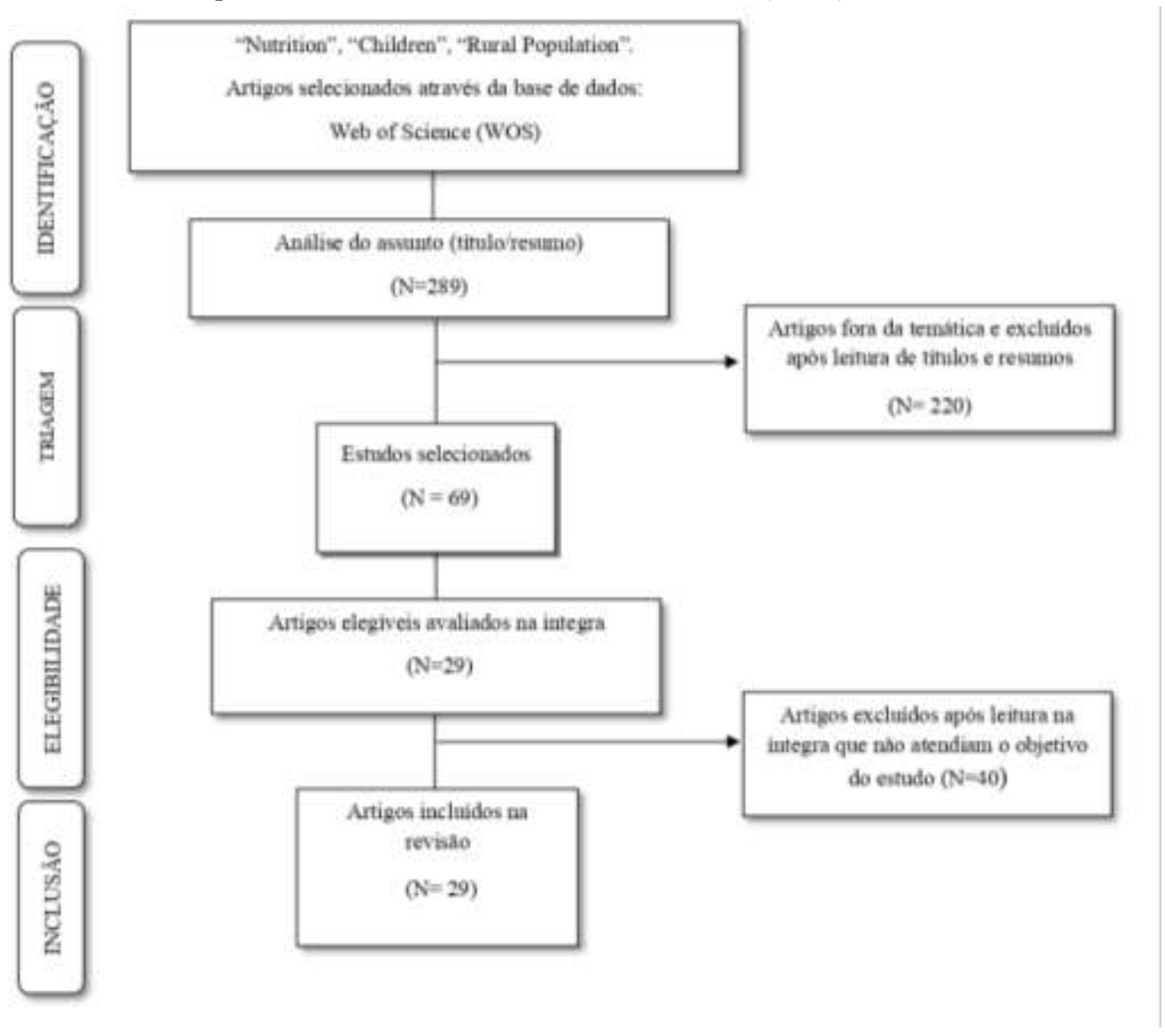

Fonte: Elaborados pelos autores.

De modo a assegurar a confiabilidade das etapas da revisão e evitar viés de seleção, as referências foram perpassadas pela estratégia procedimental a dupla checagem, com atuação independente. Onde as informações coletadas foram organizadas e compiladas eletronicamente em planilha do programa Microsoft Office Excel@versão2013.

Assim, a amostra final foi composta por 29 referências.

Para caracterização dos dados, utilizou-se um instrumento elaborado pelos autores, agrupados por similaridade de conteúdo, contendo as variáveis autoria, título, tipo de estudo, variáveis do estudo, amostra(n), pais do estudo, resultados. Permitindo, assim, sumarizar e comparar os resultados obtidos, seguindo-se o processo de análise interpretativa, a partir da literatura científica.

Em virtude de o objeto da pesquisa não englobar participação de seres humanos de forma direta ou indireta, valendose unicamente de dados bibliográficos de domínio público, justifica-se, desse modo, a dispensa de submissão à Plataforma Brasil para apreciação pelo Comitê de Ética em Pesquisa.

\section{Resultados}

A apresentação dos resultados desta revisão está sumarizada na Tabela 1.

Segue abaixo, tabela com compilado das informações encontradas nos estudos incluídos na amostra final. 
Research, Society and Development, v. 11, n. 1, e53011125276, 2022

(CC BY 4.0) | ISSN 2525-3409 | DOI: http://dx.doi.org/10.33448/rsd-v11i1.25276

Tabela 1. Artigos selecionados

\begin{tabular}{|c|c|c|c|c|c|c|}
\hline Autores & Título & Tipo do estudo & Variáveis & $\begin{array}{c}\text { Amostra } \\
\text { (n) }\end{array}$ & $\begin{array}{l}\text { País do } \\
\text { estudo }\end{array}$ & Resultados \\
\hline Samdarshi et al., 2020 & $\begin{array}{l}\text { Nutritional Status of One to Five Year Old } \\
\text { Children in Rural Haryana: A Community } \\
\text { Based Study }\end{array}$ & Quantitativo & $\begin{array}{l}\text { Peso, altura, sexo, idade. } \\
\text { Faixa etária: } 12-59 \text { meses }\end{array}$ & 1.032 & Índia & $\begin{array}{l}21,5 \% \text { das crianças com baixo peso, } 30,2 \% \text { com nanismo } \\
\text { e } 8,9 \% \text { com definhamento. }\end{array}$ \\
\hline Lukwa et al., 2020 & $\begin{array}{l}\text { Socioeconomic inequalities in food insecurity } \\
\text { and malnutrition among underfive children } \\
\text { within and between-group inequalities in } \\
\text { Zimbabwe }\end{array}$ & Quantitativo & $\begin{array}{l}\text { Peso, sexo, idade. } \\
\text { Faixa etária: } 0-59 \text { meses }\end{array}$ & 5549 & África & $\begin{array}{l}\text { A desnutrição em 2010/11 apresentou percentual de } \\
3,73 \% \text {. Em 2015, } 4,76 \% \text {. A prevalência da desnutrição na } \\
\text { zona rural foi de } 72.30 \% \text { (2010/11) e } 82.53 \% \text { (2015). }\end{array}$ \\
\hline $\begin{array}{l}\text { Nepali; } \quad \text { Simkhada; } \\
\text { Davies, } 2020\end{array}$ & $\begin{array}{lccc}\text { Association } & \text { between } & \text { wasting } & \text { and } \\
\text { foodinsecurity } & \begin{array}{c}\text { among } \\
\text { children under five }\end{array} \\
\text { years:findings } & \text { from } & \text { Nepal demographic } \\
\text { healthsurvey 2016 } & & & \\
\end{array}$ & Quantitativo & $\begin{array}{l}\text { Peso, altura, idade. } \\
\text { Faixa etária: } 0-59 \text { meses }\end{array}$ & 2.414 & Nepal & $\begin{array}{l}\text { As maiores proporções de crianças com definhamento } \\
\text { ocorreram em áreas rurais, nascidas de mães sem } \\
\text { escolaridade. }\end{array}$ \\
\hline Mahmood et al., 2020 & $\begin{array}{l}\text { Why under five children are stunted in } \\
\text { Pakistan? A multilevel analysis of Punjab } \\
\text { Multiple indicator Cluster Survey (MICS-2014) }\end{array}$ & Quantitativo & $\begin{array}{l}\text { Peso, altura, idade. } \\
\text { Faixa etária: }<5 \text { anos }\end{array}$ & 25.067 & Paquistão & $\begin{array}{l}\text { Prevalência de baixa estatura moderada em cerca de } 27 \% \\
\text { baixa estatura severa em } 10 \% \text { das crianças. } 30 \% \text { que vive } \\
\text { em áreas rurais são moderadamente raquíticas em relação } \\
\text { a } 21 \% \text { nas áreas urbanas. } 12 \% \text { são gravemente atrofiadas } \\
\text { em comparação com } 7 \% \text { nas áreas urbanas. }\end{array}$ \\
\hline Prost et al., 2019 & $\begin{array}{l}\text { Mortality and recovery following moderate and } \\
\text { severe acute malnutrition in children aged 6-18 } \\
\text { months in rural Jharkhand and Odisha, eastern } \\
\text { India: A cohort study }\end{array}$ & Quantitativo & $\begin{array}{l}\text { Peso, altura, circunferência de } \\
\text { braço, idade. } \\
\text { Faixa etária: 6-18meses }\end{array}$ & 2.869 & Índia & $\begin{array}{l}\text { Observada alta incidência de desnutrição aguda moderada } \\
\text { e de desnutrição aguda grave. }\end{array}$ \\
\hline $\begin{array}{l}\text { Gatica-Domínguez; } \\
\text { Barros, } 2019\end{array}$ & $\begin{array}{l}\text { Ethnic inequalities and trends in stunting } \\
\text { prevalence among Guatemalan children: an } \\
\text { analysis using national health surveys 1995- } \\
2014\end{array}$ & Quantitativo & $\begin{array}{l}\text { Idade, altura. } \\
\text { Faixa etária: }<5 \text { anos }\end{array}$ & 37.020 & Guatemala & $\begin{array}{l}\text { As crianças nas áreas rurais apresentaram } 53 \% \text { de excesso } \\
\text { de atrofia em comparação com as zonas urbanas. As } \\
\text { crianças indígenas residentes nas zonas rurais estão mais } \\
\text { vulneráveis às iniquidades nutricionais. A taxa de } \\
\text { Nanismo reduziu. }\end{array}$ \\
\hline Mark et al., 2019 & $\begin{array}{l}\text { Nutritional status and disease severity } \\
\text { inchildren acutely presenting to a primaryhealth } \\
\text { clinic in rural Gambia }\end{array}$ & Quantitativo & $\begin{array}{c}\text { Idade, altura, peso. } \\
\text { Faixa etária: }<5 \text { anos } \\
\end{array}$ & 5.021 & Gâmbia & $\begin{array}{l}\text { Crianças > } 12 \text { meses apresentaram maior prevalência de } \\
\text { nanismo leve, moderado e grave. A prevalência de } \\
\text { definhamento foi semelhante entre as faixas etárias } \\
\text { consideradas. }\end{array}$ \\
\hline $\begin{array}{l}\text { Wilchez; } \quad \text { Sánchez; } \\
\text { Diaz, } 2019\end{array}$ & $\begin{array}{l}\text { Knowledge, attitudes and food practices in } \\
\text { caregivers and nutritional status in infants from } \\
\text { Ventaquemada, Boyacá, Colombia }\end{array}$ & Misto & $\begin{array}{c}\text { Idade, peso, altura, IMC, } \\
\text { circunferência cefálica para a } \\
\text { idade e circunferência do braço } \\
\text { médio. } \\
\text { Faixa etária: }<24 \text { meses }\end{array}$ & 170 & Colômbia & $\begin{array}{l}\text { Foi identificada na amostra presença de desnutrição aguda } \\
\text { moderada, excesso de peso e obesidade, baixa estatura e } \\
\text { desnutrição global. }\end{array}$ \\
\hline $\begin{array}{l}\text { Jager; } \quad \text { Giller; } \\
\text { Brouwer, } 2018\end{array}$ & $\begin{array}{l}\text { Food and nutrient gaps in rural northern ghana: } \\
\text { does production of smallholder farming } \\
\text { households support adoption of food-based } \\
\text { dietary guidelines? }\end{array}$ & Quantitativo & $\begin{array}{l}\text { Idade, peso, comprimento, IMC. } \\
\text { Faixa etária: } 6-23 \text { meses }\end{array}$ & 329 & Gana & $40 \%$ das crianças eram raquíticas. \\
\hline Headey et al., 2018 & $\begin{array}{l}\text { Remoteness,urbanizati And child nutrition in } \\
\text { Sub-saharan Africa }\end{array}$ & Quantitativo & $\begin{array}{c}\text { Idade, altura } \\
\text { Faixa etária: } 24-59 \text { meses }\end{array}$ & 74.398 & $\begin{array}{c}\text { África } \\
\text { Subsaariana }\end{array}$ & $\begin{array}{l}\text { A desnutrição é substancialmente maior nas áreas rurais, } \\
\text { em comparação com as áreas urbanas. }\end{array}$ \\
\hline Sapunar et al., 2018 & $\begin{array}{l}\text { High prevalence of Overweight, Obesity, } \\
\text { Insulin resistance and Metabolic syndrome in } \\
\text { Rural Children and Adolescents }\end{array}$ & Quantitativo & $\begin{array}{l}\text { Idade, peso, IMC. } \\
\text { Faixa etária: } 10,4 \pm 1,0 \text { anos }\end{array}$ & 208 & Chile & $\begin{array}{l}71 \% \text { dos escolares apresentavam distúrbios nutricionais } \\
\text { por excesso de peso ( } 37,9 \% \text { com sobrepeso e } 33,1 \% \text { com } \\
\text { obesidade). }\end{array}$ \\
\hline
\end{tabular}


Research, Society and Development, v. 11, n. 1, e53011125276, 2022

(CC BY 4.0) | ISSN 2525-3409 | DOI: http://dx.doi.org/10.33448/rsd-v11i1.25276

\begin{tabular}{|c|c|c|c|c|c|c|}
\hline Cortes et al., 2018 & $\begin{array}{l}\text { Poor breastfeeding, complementary feeding and } \\
\text { dietary diversity in children and their relationship } \\
\text { with stunting in rural communities }\end{array}$ & Misto & $\begin{array}{l}\text { Idade, altura, alimentação } \\
\text { complementar e diversidade } \\
\text { alimentar mínima. } \\
\text { Faixa etária: } 1-24 \text { meses }\end{array}$ & 189 & México & A baixa estatura foi encontrada em $10,1 \%$ da amostra. \\
\hline $\begin{array}{l}\text { Gizaw; Woldu; Bitew, } \\
2018\end{array}$ & $\begin{array}{l}\text { Acute malnutrition among children aged6-59 } \\
\text { months of the nomadic population inhadaleala } \\
\text { district, afar region, northeastethiopia }\end{array}$ & Quantitativo & $\begin{array}{l}\text { Idade; Circunferência média do } \\
\text { braço. } \\
\text { Faixa etária: } 6-59 \text { meses. }\end{array}$ & 591 & Etiópia & A prevalência de desnutrição aguda foi de $11,8 \%$. \\
\hline Samiak; Emeto, 2017 & $\begin{array}{l}\text { Vaccination and nutritional status of children in } \\
\text { karawari, east sepik province, papua new guinea }\end{array}$ & Quantitativo & $\begin{array}{l}\text { Idade, altura, peso. } \\
\text { Faixa etária: }<5 \text { anos }\end{array}$ & 105 & $\begin{array}{l}\text { Papua Nova } \\
\text { Guiné }\end{array}$ & $\begin{array}{l}\text { A prevalência geral de desnutrição aguda foi } 15,8 \% \text {. A } \\
\text { incidência de baixo peso foi de } 26 \% \text {. A prevalência geral } \\
\text { de nanismo foi de } 45,3 \% \text {. }\end{array}$ \\
\hline Raymond et al., 2017 & $\begin{array}{l}\text { Analysis of nutritional adequacy of local foods } \\
\text { for meeting dietary requirements of children aged } \\
6-23 \text { months in rural central tanzania }\end{array}$ & Quantitativo & $\begin{array}{l}\text { Idade, peso, altura. } \\
\text { Faixa etária: } 6-23 \text { meses }\end{array}$ & 400 & Tanzânia & $\begin{array}{l}\text { A prevalência de baixa estatura e baixo peso foi de } 30 \text { a } \\
41 \% \text { e } 4 \text { a } 9 \% \text {, respectivamente. }\end{array}$ \\
\hline $\begin{array}{l}\text { Barrantes-Montoya; } \\
\text { Calderón-Rodríguez; } \\
\text { Fernández-Rojas, } \\
2017\end{array}$ & $\begin{array}{l}\text { Prevalence of overweight, obesity and associated } \\
\text { factors in preschoolers in the canton of turrialba }\end{array}$ & Quantitativo & $\begin{array}{c}\text { Idade, peso, altura, IMC. } \\
\text { Faixa etária: } 3 \text { Anos e } 6 \text { meses, } 7 \\
\text { anos e } 8 \text { meses }\end{array}$ & 643 & Costa Rica & $\begin{array}{l}\text { Na zona rural houve um menor percentual de crianças } \\
\text { abaixo do peso (5\%) em comparação com as crianças das } \\
\text { áreas urbanas (8\%). O excesso de peso (sobrepeso ou } \\
\text { obesidade) para a zona rural foi de } 19 \% \text {, para a área } \\
\text { urbana foi } 18 \% \text {. A baixa estatura foi maior na zona rural } \\
(8 \%) \text { em comparação com a zona urbana (3\%). }\end{array}$ \\
\hline Ochoa, 2017 & $\begin{array}{l}\text { Evaluation of the nutritional status of children } \\
\text { under } 5 \text { years of age: concordance between } \\
\text { anthropometric indices in the indigenous } \\
\text { population of chiapas (mexico) }\end{array}$ & Quantitativo & $\begin{array}{l}\text { Idade, sexo, peso e altura } \\
\text { Faixa etária: }<5 \text { anos }\end{array}$ & 1.600 & México & $\begin{array}{l}\text { IMC apresentou baixa prevalência de desnutrição e maior } \\
\text { prevalência de sobrepeso e obesidade. }\end{array}$ \\
\hline Belesova et al., 2017 & $\begin{array}{l}\text { Household cereal crop harvest and children's } \\
\text { nutritional status in rural burkina faso }\end{array}$ & Quantitativo & $\begin{array}{c}\text { Idade, circunferência do braço } \\
\text { médio. } \\
\text { Faixa etária: }<5 \text { anos } \\
\end{array}$ & 975 & Burkina Faso & $\begin{array}{l}14 \% \text { das crianças apresentaram indicativos de desnutrição } \\
\text { aguda. }\end{array}$ \\
\hline $\begin{array}{lr}\text { Bergel } & \text { Sanchís; } \\
\text { Cesani; } & \text { Oyhenart. } \\
2017 & \\
\end{array}$ & $\begin{array}{l}\text { Malnutricion infantil e inseguridad alimentaria } \\
\text { como expresión de las condiciones socio- } \\
\text { económicas familiares en villaguay, argentina } \\
(2010-2012) \text {. Un enfoque biocultural }\end{array}$ & Quantitativo & $\begin{array}{l}\text { Idade, peso, altura. } \\
\text { Faixa etária: }<7 \text { anos }\end{array}$ & 303 & Argentina & $\begin{array}{l}28,7 \% \text { das crianças apresentavam algum tipo de } \\
\text { desnutrição. A prevalência de desnutrição foi menor na } \\
\text { zona rural, em comparação com as áreas periurbana e } \\
\text { urbana. }\end{array}$ \\
\hline Juma et al., 2016 & $\begin{array}{l}\text { Prevalence and assessment of malnutrition } \\
\text { among children attending the reproductive and } \\
\text { child health clinic at bagamoyo district hospital, } \\
\text { tanzania }\end{array}$ & Quantitativo & $\begin{array}{c}\text { Idade, peso, altura, } \\
\text { circunferência do Braço Médio. } \\
\text { Faixa etária: }<5 \text { anos }\end{array}$ & 63.237 & Tanzânia & $\begin{array}{l}\text { A proporção geral de nanismo e de baixo peso foi de } 8,37 \\
\text { e } 5,74 \text {. As crianças das áreas rurais apresentaram taxas } \\
\text { maiores de nanismo e baixo peso, em comparação com as } \\
\text { crianças das áreas urbanas. }\end{array}$ \\
\hline Sharaf; rashad., 2016 & $\begin{array}{l}\text { Regional inequalities in child malnutrition in } \\
\text { egypt, jordan, and yemen: a blinder-oaxaca } \\
\text { decomposition analysis }\end{array}$ & Quantitativo & $\begin{array}{l}\text { Idade, altura. } \\
\text { Faixa etária: }<5 \text { anos }\end{array}$ & 31.017 & $\begin{array}{l}\text { Egito, } \\
\text { Jordânia e } \\
\text { Iêmen }\end{array}$ & $\begin{array}{l}\text { Taxas de nanismo: } 20 \% \text { no Egito, } 46,5 \% \text { no lêmen e } 7,7 \% \\
\text { na Jordânia. A disparidade rural-urbana na desnutrição } \\
\text { infantil foi menor no caso do Egito }(2,3 \%) \text { e Jordânia } \\
(1,5 \%) \text { enquanto a disparidade regional foi significativa } \\
\text { no caso do lêmen }(17,7 \%) \text {. }\end{array}$ \\
\hline Luo et al., 2015 & $\begin{array}{l}\text { Micronutrient deficiencies and developmental } \\
\text { delays among infants: evidence from a cross- } \\
\text { sectional survey in rural china }\end{array}$ & Quantitativo & $\begin{array}{l}\text { Idade, peso, altura. } \\
\text { Faixa etária: } 6-12 \text { meses }\end{array}$ & 1.808 & China & $\begin{array}{l}3,7 \% \text { tinham baixa estatura, } 1,2 \% \text { estavam abaixo do peso } \\
\text { e } 1,6 \% \text { tinham magreza. }\end{array}$ \\
\hline Hanieh et al., 2015 & $\begin{array}{l}\text { Antenatal and early infant predictors of postnatal } \\
\text { growth in rural vietnam: a prospective cohort } \\
\text { study }\end{array}$ & Quantitativo & $\begin{array}{l}\text { Idade, comprimento, peso. } \\
\text { Faixa etária: } 6 \text { meses. }\end{array}$ & 1.046 & Vietnã & A prevalência de nanismo foi de $6,4 \%$. \\
\hline Ochoa, 2015 & Nutritional status of two generations of brothers & Quantitativo & Idade, peso, altura, IMC. & 222 & México & $43,4 \%$ dos irmãos e irmãs avaliados em 2010-2011 \\
\hline
\end{tabular}


Research, Society and Development, v. 11, n. 1, e53011125276, 2022

(CC BY 4.0) | ISSN 2525-3409 | DOI: http://dx.doi.org/10.33448/rsd-v11i1.25276

\begin{tabular}{|c|c|c|c|c|c|c|}
\hline & $\begin{array}{l}\text { and sisters }<5 \text { years of age beneficiaries from } \\
\text { opportunities living in marginalized rural } \\
\text { communities in chiapas, mexico }\end{array}$ & & Faixa etária: $<5$ anos & & & $\begin{array}{l}\text { apresentaram retardo de crescimento, a prevalência de } \\
\text { baixo peso diminuiu de } 18 \% \text { para } 13,2 \% \text {. O sobrepeso e a } \\
\text { obesidade aumentaram de } 24,8 \% \text { em 2002-2003 para } \\
36,8 \% \text { em 2010-2011. }\end{array}$ \\
\hline Veghari et al., 2015 & $\begin{array}{l}\text { The comparison of under-5-year nutritional status } \\
\text { among fars-native, turkman and sistani ethnic } \\
\text { groups in the north of iran }\end{array}$ & Quantitativo & $\begin{array}{l}\text { Idade, peso, altura. } \\
\text { Faixa etária: }<5 \text { anos }\end{array}$ & 2.530 & Irã & $\begin{array}{l}\text { Desnutrição em } 6,6 \%, 18,5 \% \text { e } 3,3 \% \text { com base no baixo } \\
\text { peso, baixa estatura e magreza. }\end{array}$ \\
\hline $\begin{array}{l}\text { Nuruddin; Hadden., } \\
2015\end{array}$ & $\begin{array}{l}\text { Are pre-school girls more likely to be under- } \\
\text { nourished in rural thatta, pakistan?-a cross- } \\
\text { sectional study }\end{array}$ & Quantitativo & $\begin{array}{l}\text { Idade, peso, altura. } \\
\text { Faixa etária: } 0-35 \text { meses }\end{array}$ & 1.051 & Paquistão & $\begin{array}{l}\text { Uma grande proporção de crianças apresentou baixa } \\
\text { estatura }(52,9 \%) \text {, emagrecimento }(22,9 \%) \text { e baixo peso } \\
(46,5 \%) \text {. }\end{array}$ \\
\hline Galetti et al., 2016 & $\begin{array}{l}\text { Rural beninese children are at risk of zinc } \\
\text { deficiency according to stunting prevalence and } \\
\text { plasma zinc concentration but not dietary zinc } \\
\text { intakes }\end{array}$ & Quantitativo & $\begin{array}{l}\text { Idade, peso, altura, e } \\
\text { Circunferência Média do Braço. } \\
\text { Faixa etária: }<5 \text { anos }\end{array}$ & 273 & Benin & A baixa estatura foi de $51 \%$ (139 de 273 ). \\
\hline $\begin{array}{l}\text { García-Parra et al., } \\
2016\end{array}$ & $\begin{array}{l}\text { Are there changes in the nutritional status of } \\
\text { children of oportunidades families in rural } \\
\text { chiapas, mexico? A cohort prospective study }\end{array}$ & Quantitativo & $\begin{array}{l}\text { Idade, peso, altura. } \\
\text { Faixa etária: }<5 \text { anos }\end{array}$ & 222 & México & Alta prevalência de déficit de estatura $(40,1 \%)$. \\
\hline Yadav et al., 2016 & $\begin{array}{l}\text { An epidemiological study of malnutrition among } \\
\text { under five children of rural and urban haryana }\end{array}$ & Quantitativo & $\begin{array}{l}\text { Idade, peso, altura. } \\
\text { Faixa etária: } 3-60 \text { meses. }\end{array}$ & 750 & India & $\begin{array}{l}41,3 \% \text { das crianças estavam abaixo do peso e } 14 \% \\
\text { apresentavam baixo peso grave. A prevalência de baixo } \\
\text { peso e baixo peso grave foi maior na área rural, em } \\
\text { comparação com a área urbana. }\end{array}$ \\
\hline
\end{tabular}

Fonte: Elaborados pelos autores. 
A partir das informações expressas no Quadro 1, observamos que foram incluídos estudos de 26 países, sendo representados na amostra: Índia, África, Nepal, Paquistão, Guatemala, Gâmbia, Colômbia, Gana, África Subsaariana, Chile, México, Etiópia, Papua Nova Guiné, Tanzânia, Costa Rica, Burkisa Faso, Argentina, Egito, Jordânia, Iemên, China, Vietnã, México, Irã, Paquistão e Benin. Se sobressaindo as referências dos países do México (4), Paquistão (2), índia (2), e Tanzânia (2).

As principais variáveis consideradas para os estudos incluídos na amostra final foram: idade, peso, altura e circunferência média do braço. Em relação ao estado nutricional, frente ao levantamento realizado, ficou evidenciado que o baixo peso foi o estado nutricional predominante nas crianças que viviam em comunidades rurais, como também, a prevalência do nanismo e da magreza, indicando aspectos sensíveis relativos à vulnerabilidade nutricional.

\section{Discussão}

Por meio desta revisão, observou-se que estado nutricional de crianças residentes em comunidade rurais evidenciouse que o nanismo (importante indicador de desnutrição) constatou-se como um dos principais achados, variou entre 3,7\% e 53\% das crianças nos estudos realizados na China, Vietnã, México, Benin, Paquistão, Papua Nova Guiné e Guatemala (Luo et al., 2015; Hanieh et al., 2015; Ochoa, 2015; Galetti et al., 2016; Nuruddin, \& Hadden, 2015; Samiak \& Emeto, 2017; GaticaDomínguez et al., 2019).

Guatemala, a prevalência geral de nanismo diminuiu 9,8 pontos percentuais de 1995 a 2014 (Gatica-Domínguez et al., 2019). Em um outro estudo, realizado na África, a desnutrição em 2010/11 apresentou percentual de 3,73\% e em 2015, 4,76\%. Já a prevalência da desnutrição na zona rural foi de 72.30\% (2010/11) e 82.53\% (2015), revelando um aumento significativo no percentual (Lukwa et al., 2010)]. No Brasil, o déficit de altura caiu de 29,3\% (1974-75) para 7,2\% (2008-09) entre meninos e de $26,7 \%$ para $6,3 \%$ nas meninas, mas se sobressaiu no meio rural da região Norte: $16 \%$ dos meninos e 13,5\% das meninas (BRASIL, 2010).

Quanto ao baixo peso apresentou em mais de $40 \%$ das crianças nas pesquisas dos países da Índia, Tânzania e Paquistão (Nuruddin, \& Hadden, 2015; Prost et al.,2019; Raymond et al., 2017), sendo semelhante a um estudo realizado em Bangladash, o qual indicou a prevalência de 37,7\% das crianças com baixo peso (Ali et al., 2019). No entanto, estudos realizados no Chile e no México, apontam baixa prevalência de desnutrição e maior prevalência de sobrepeso e obesidade (Sapunar et al., 2018; Ochoa, 2017). Assim como, em uma pesquisa nos EUA, os resultados apontaram que 51\% das crianças apresentavam diagnóstico de sobrepeso ou obesidade de acordo com o IMC para idade (Kaiser et al., 2015).

No que concerne à magreza (emaciação, emagrecimento, definhamento ou desnutrição aguda), a China foi o país com o menor percentual de crianças com esse indicador de desnutrição, 1,6\% (Luo et al., 2015). Porém, no Paquistão, 22,9\% das crianças estudadas apresentaram o maior percentual de magreza (Nuruddin, \& Hadden, 2015). O Iêmen também apresentou uma porcentagem importante de 17,7\% das crianças avaliadas (Sharaf \& Rashad, 2016). Em 2020, Agências das Nações Unidas (UNICEF) fizeram um alerta apontando que as crianças iemenitas sofrem desnutrição infantil aguda sem precedentes e que uma em cada quatro crianças têm desnutrição aguda em algumas áreas do país que sofrem os efeitos do conflito e do colapso econômico (ONU, 2020).

Para a avaliação do estado nutricional, a maioria dos estudos utilizaram os padrões e valores de crescimento da criança: peso - altura (P / E), altura - idade (A / I), peso - idade (P / I) e índice de massa corporal (IMC), segundo recomendações da Organização Mundial da Saúde (OMS) (WHO, 2006; Onis, 2017).

O emprego dos indicadores antropométricos na avaliação do estado nutricional de indivíduo ou coletividades é, entre várias opções, a mais apropriada e viável para ser aplicável em serviços de saúde. Além de ter em consideração as suas vantagens como: baixo custo, simplicidade de realização, facilidade na aplicação e padronização, amplitude dos aspectos 
analisados, não é invasiva. Assim como, ser uma ferramenta importante na investigação em nutrição e aplicável em todas as fases do curso da vida. Por serem usados mundialmente, esses indicadores permitem que se façam comparações internacionais da situação nutricional de grupos vulneráveis e o vasto estudo de seus determinantes em plano regional, nacional ou internacional (Brasil, 2011)

A circunferência do braço médio (CA), também, foi um utilizado em alguns dos estudos, como indicador de risco em crianças com desnutrição aguda grave, pois oferece grande precisão no que diz respeito à estimativa das reservas calóricas e da massa muscular (Chiabi et al., 2017; Rodríguez Wilchez et al., 2019).

Diante o exposto, a nutrição e a segurança alimentar em crianças dependem fortemente de fatores socioeconômicos (Lukwa et al., 2020). Essa alta prevalência de insegurança alimentar observada em alguns países pode ser um dos fatores subjacentes para a alta ocorrência de diferentes formas de subnutrição, como nanismo, emagrecimento e baixo peso. Alguns estudos apontaram que crianças com mães e pais não-escolarizados, falta de instalações sanitárias e baixa renda estão significativamente associados à probabilidade de nanismo moderado e grave (Nepali et al., 2020; Mahmood et al.,2020).

Nos achados_uma análise multivariável constatou que as crianças cujas mães tinham maior nível de escolaridade e eram trabalhadoras qualificadas tiveram o escore de diversidade alimentar (DDS) mais alto (15\% e 48\% respectivamente) em comparação com suas contrapartes. A diversidade alimentar mostrou forte associação positiva com o status de renda familiar (Ali et al., 2019).

Em outras pesquisas, a prevalência de desnutrição ocorreu nas comunidades urbanas e não nas comunidades rurais e com o excesso de peso de $1 \%$ maior na zona rural do que na urbana. Nesse sentido, considera-se que o mercado encontrou uma alternativa de consumo específico nas populações mais pobres a quem se oferece comida barata, com alta densidade calórica, mas nutricionalmente deficiente (Barrantes-Montoya et al., 2017; Bergel et al., 2017).

Em uma pesquisa realizada para avaliar a evolução do estado nutricional de crianças beneficiárias de um Programa Social por 9 anos, mostrou que apesar dessas famílias terem melhorado suas condições de vida, as crianças ainda apresentavam alta prevalência de déficit de crescimento $(40,1 \%)$ e 69,6\% ainda não haviam se recuperado. Crianças que foram inicialmente diagnosticadas com estado nutricional normal e tornaram-se raquíticas 2 anos depois, apresentaram um risco maior de continuar com baixa estatura na idade escolar e na adolescência. Isso pode ser resultado de um processo de adaptação alcançado por meio de uma redução da velocidade de crescimento (García-Parra et al., 2016).

Considerando o impacto da pobreza sobre a desnutrição, o setor da saúde é essencial para atenuar esse sofrimento por meio do fornecimento de serviços básicos de saúde (Lukwa et al., 2020). Entre as crianças pobres das áreas rurais, deve-se prestar atenção especial à distribuição igualitária dos serviços públicos nas regiões para aumentar as chances justas de saúde infantil, na qual, uma nutrição infantil adequada é indispensável para o desenvolvimento físico e cognitivo adequado das crianças (Akombi et al., 2019).

Deste modo, políticas que promovam a disponibilidade, acesso e consumo de diversos alimentos ricos em nutrientes precisam ser incentivadas, especialmente para os grupos vulneráveis onde tais alimentos têm o potencial de reduzir os impactos da insegurança alimentar (Nepali et al., 2020). Assim a inclusão de Planejamento e Serviços de Saúde de Base Comunitária; Programas de alimentação suplementar e educação em saúde e nutrição em comunidades carentes, são essências principalmente em comunidades rurais, por meio de voluntários de saúde da comunidade (Lukwa et al., 2020).

No Nepal, além de intervenções que abordavam a insegurança alimentar, intervenções integradas foram implementadas no país após o lançamento do Plano Multissetorial de Nutrição I e II, o qual trata sobre educação, saneamento, comportamento alimentar de subgrupos da população, como meninas adolescentes, mulheres grávidas e lactantes e seus bebês entre os grupos rurais e mais pobres (Field, 1987). 
No Brasil, foi criada a Lei Orgânica de Segurança Alimentar e Nutricional (LOSAN) que surge pelo dever do Estado em respeitar, proteger, promover e fiscalizar a realização do direito humano à alimentação adequada, bem como garantir os mecanismos para sua exigibilidade, com acesso regular e permanente a alimentos de qualidade, em quantidade suficiente, sem comprometer o acesso à outras necessidades essenciais (Brasil, 2006).

A Atenção Primária à Saúde (APS) identificada como uma estratégia primordial no combate à disparidade da assistência à saúde no Brasil, representa o contato preferencial dos usuários com os sistemas de saúde, e desenvolve suas ações através de técnicas educativas que possibilitam as intervenções no processo de saúde-doença da população. Nesse sentido, a alimentação e a nutrição fazem parte das condições básicas para a promoção e proteção à saúde, sendo que as estratégias de Educação Alimentar e Nutricional (EAN) constituem papel fundamental no contexto da Atenção Básica e, em especial, na Estratégia Saúde da Família (ESF), que surgiu para reorganizar a rede de atenção e aproximar o trabalho educativo na comunidade, expandindo o seu campo de intervenções (Alencar et al., 2016; Oliveira \& Pereira, 2013).

Ainda no Brasil, outra contribuição à segurança alimentar no país, foi a inserção do profissional nutricionista na ESF que se dedica, entre outras práticas, à promoção de uma alimentação saudável, sendo importante ator na luta pela garantia da segurança alimentar e nutricional (SAN) (Brasil, 2008). Nessa perspectiva, salienta-se que participação dos nutricionistas em programas de residência multiprofissionais contribui sistematicamente para o direcionamento de ações com vistas à integralidade do cuidado, tendo ainda, a promoção da saúde como ferramenta poderosa.

\section{Conclusão}

O estado nutricional de desnutrição foi apontado na maioria dos estudos, indicando que as crianças em comunidades rurais, com pais não escolarizados, em condições inadequadas de moradia, vivendo em zonas de conflito e vulnerabilidade socioeconômica encontram-se mais susceptíveis à insegurança alimentar infantil. Todavia, uma transição positiva em alguns países, com indicadores mais otimistas e promissores, pôde ser observada.

Além desse fator, é relevante apontar que mudanças nos padrões de consumo de alimentos podem induzir a outro fenômeno, notadamente excesso de peso e obesidade na infância em comunidades rurais, destacando a imprescindibilidade de ações de intervenção para controle e tratamento. Assim como também atividades com foco para educação e promoção da saúde voltadas para o estímulo a um comportamento alimentar saudável, todas com execução possível a nível de Atenção Primária à Saúde.

Portanto, os resultados com a implementação de políticas públicas bem articuladas delineadas décadas atrás já são tangíveis e evidenciam que avanços foram concretizados no campo da segurança alimentar para crianças. Contudo, ainda persistem iniquidades evitáveis em saúde, que atingem majoritariamente os mais vulneráveis, conforme evidenciado pelo presente estudo.

Nesse sentido, salienta-se a relevância de políticas públicas bem estruturadas e sistematicamente efetivas de inclusão social, no combate à pobreza e com foco para a redução da insegurança alimentar, visando promover a disponibilidade, o acesso e o consumo de alimentos ricos em nutrientes.

\section{Referências}

Alencar, A. P.A, Lustoza Xavier, S. P., Agostinho da Silva Laurentino, P., Frazão Lira, P., Barbosa do Nascimento, V., Carneiro, N., \& Affonso Fonseca, F. L. (2016). Impacto do programa mais médicos na atenção básica de um município do sertão central nordestino. Gestão e Sociedade, 10(26), 1290. https://doi.org/10.21171/ges.v10i26.2085

Almeida, J. A., Santos, A. S., Nascimento, M. A. de O., Oliveira, J. V. C., Silva, D. G. da, Mendes-Netto, R. S., \& Embrapa Tabuleiros Costeiros, Brasil. (2017). Fatores associados ao risco de insegurança alimentar e nutricional em famílias de assentamentos rurais. Ciência \& Saúde Coletiva, 22(2), 479-488. https://doi.org/10.1590/1413-81232017222.27102015 
Ali, N. B., Tahsina, T., Hoque, D. Md. E., Hasan, M. M., Iqbal, A., Huda, T. M., \& El Arifeen, S. (2019). Association of food security and other socioeconomic factors with dietary diversity and nutritional statuses of children aged 6-59 months in rural Bangladesh. Plos One, 14(8), e0221929. https://doi.org/10.1371/journal.pone.0221929

Akombi, B. J., Agho, K. E., Renzaho, A. M., Hall, J. J., \& Merom, D. R. (2019). Trends in socioeconomic inequalities in child undernutrition: Evidence from Nigeria Demographic and Health Survey (2003 - 2013). Plos One, 14(2), e0211883. https://doi.org/10.1371/journal.pone.0211883

Brasil (18 set 2006). Lei n 11.346 de 15 set. 2006. Lei Orgânica de Segurança Alimentar e Nutricional. Cria o Sistema Nacional de Segurança Alimentar e Nutricional - SISAN com vistas em assegurar o direito humano à alimentação adequada e dá outras providências. Diário Oficial da União. http://www.planalto.gov.br/ccivil_03/_ato2004-2006/2006/lei/111346.htm

Brasil (2008). Conselho Nacional de Segurança Alimentar e Nutricional. O nutricionista e a segurança alimentar. Brasília, DF. https://bvsms.saude.gov.br/bvs/publicacoes/politica_nacional_alimentacao_nutricao.pdf

Brasil, Ministério da Saúde (2020). Criança e Adolescente. Saúde de A a Z. Brasília, DF; Ministério da Saúde. https://bvsms.saude.gov.br/bvs/publicacoes/sus_az_garantindo_saude_municipios_3ed_p1.pdf

Brasil, Ministério da Saúde (2019). Portal de Boas Práticas em Saúde da Mulher, da Criança e do Adolescente. Atlas of Childhood Obesity. https://portaldeboaspraticas.iff.fiocruz.br/

Brasil. Ministério da Saúde (2015). Ministério cria política de atenção à saúde da criança. Brasília, DF; Ministério da Saúde. https://portaldeboaspraticas.iff.fiocruz.br/wp-content/uploads/2018/07/Pol\%C3\%ADtica-Nacional-de-Aten\%C3\%A7\%C3\%A3o-Integral-\%C3\%A0Sa\%C3\%BAde-da-Crian\%C3\%A7a-PNAISC-Vers\%C3\%A3o-Eletr\%C3\%B4nica.pdf

Brasil. Ministério da Saúde (2008). Secretaria de Atenção à Saúde. Departamento de Atenção Básica. Protocolos do Sistema de Vigilância Alimentar e Nutricional - SISVAN. Brasília, DF; Ministério da Saúde. http://189.28.128.100/dab/docs/portaldab/publicacoes/protocolo_sisvan.pdf

Brasil. Ministério da Saúde (2018). Secretaria de Atenção à Saúde. Departamento de Ações Programáticas Estratégicas. Política Nacional de Atenção Integral à Saúde da Criança. Brasília, DF; Ministério da Saúde; 180. https://bvsms.saude.gov.br/bvs/saudelegis/gm/2015/prt1130_05_08_2015.html

Brasil. Ministério da Saúde (2011). Secretaria de Atenção à Saúde. Departamento de Atenção Básica. Orientações para a coleta e análise de dados antropométricos em serviços de saúde: Norma Técnica do Sistema de Vigilância Alimentar e Nutricional - SISVAN. Brasília, DF; Ministério da Saúde;76. https://bvsms.saude.gov.br/bvs/publicacoes/orientacoes_coleta_analise_dados_antropometricos.pdf

Brasil. Ministério da Saúde (2010). POF 2008-2009: desnutrição cai e peso das crianças brasileiras ultrapassa padrão internacional. Brasília, DF; Ministério da Saúde. $\quad$ https://censo2010.ibge.gov.br/noticias-censo.html?busca=pof-20082009-desnutricao-cai-peso-criancas-brasileiras-ultrapassa-padraointernacional\&idnoticia $=1699 \&$ view $=$ noticia

Brasil. Ministério da Saúde (2011). Secretaria de Atenção à Saúde. Departamento de Atenção Básica. Orientações para a coleta e análise de dados antropométricos em serviços de saúde: Norma Técnica do Sistema de Vigilância Alimentar e Nutricional - SISVAN / Ministério da Saúde, Secretaria de Atenção à Saúde, Departamento de Atenção Básica. - $\quad$ Brasília: Ministério da Saúde, $76 \quad$ p. https://bvsms.saude.gov.br/bvs/publicacoes/orientacoes_coleta_analise_dados_antropometricos.pdf

Barrantes-Montoya, A, Rodríguez, A. C., \& Rojas, X. F. (2017). Prevalencia de sobrepeso, obesidad y factores asociados en preescolares del cantón de Turrialba. Población y Salud en Mesoamérica. https://doi.org/10.15517/psm.v15i1.26278

Bergel Sanchís, M. L., Cesani, M. F., \& Oyhenart, E. E. (2016). Malnutricion infantil e inseguridad alimentaria como expresión de las condiciones socioeconómicas familiares en Villaguay, Argentina (2010-2012). Un enfoque biocultural. Población y Salud en Mesoamérica, 14(2). https://doi.org/10.15517/psm.v14i2.27305

Chiabi, A., Mbanga, C., Mah, E., Nguefack Dongmo, F., Nguefack, S., Fru, F., \& Fru, A. (2017). Weight-for-height z score and mid-upper arm circumference as predictors of mortality in children with severe acute malnutrition. Journal of Tropical Pediatrics, fmw083. https://doi.org/10.1093/tropej/fmw083

Conselho nacional de segurança alimentar(2004). Princípios e diretrizes de uma política de segurança alimentar. Brasília, DF; Editora Positiva.https://www.ipea.gov.br/participacao/images/pdfs/conferencias/Seguranca_Alimentar_II/textos_referencia_2_conferencia_seguranca_alimentar.pdf Ercole, F. F., Melo, L. S. de, \& Alcoforado, C. L. G. C. (2014). Integrative review versus systematic review. Reme: Revista Mineira de Enfermagem, 18(1). https://doi.org/10.5935/1415-2762.20140001

Field, J. O. (1987). Multisectoral nutrition planning: A post-mortem. Food Policy, 12(1), 15-28. https://doi.org/10.1016/0306-9192(87)90044-3

Food and Agriculture Organization of the United Nations(2018), United Nations, Economic Commission for Africa(ECA). Africa regional overview of food security and nutrition: addressing the threat from climate variability and extremes for food security and nutrition. https://www.fao.org/familyfarming/detail/en/c/1181810/

García-Parra, E., Ochoa-Díaz-López, H., García-Miranda, R., Moreno-Altamirano, L., Solís-Hernández, R., \& Molina-Salazar, R. (2016). Are there changes in the nutritional status of children of Oportunidades families in rural Chiapas, Mexico? A cohort prospective study. Journal of Health, Population and Nutrition, 35(1), 1. https://doi.org/10.1186/s41043-015-0038-5

Gatica-Domínguez, G., Victora, C., \& Barros, A. J. D. (2019). Ethnic inequalities and trends in stunting prevalence among Guatemalan children: An analysis using national health surveys 1995-2014. International Journal for Equity in Health, 18(1), 110. https://doi.org/10.1186/s12939-019-1016-0

Galetti, V., Mitchikpè, C. E. S., Kujinga, P., Tossou, F., Hounhouigan, D. J., Zimmermann, M. B., \& Moretti, D. (2016). Rural beninese children are at risk of zinc deficiency according to stunting prevalence and plasma zinc concentration but not dietary zinc intakes. The Journal of Nutrition, 146(1), 114-123. https://doi.org/10.3945/jn.115.216606

Hanieh, S., Ha, T. T., De Livera, A. M., Simpson, J. A., Thuy, T. T., Khuong, N. C., ... Biggs, B.-A. (2015). Antenatal and early infant predictors of postnatal growth in rural Vietnam: A prospective cohort study. Archives of Disease in Childhood, 100(2), 165-173. https://doi.org/10.1136/archdischild-2014-306328 
Kaiser, L. L., Aguilera, A. L., Horowitz, M., Lamp, C., Johns, M., Gomez-Camacho, R., \& de la Torre, A. (2015). Correlates of food patterns in young Latino children at high risk of obesity. Public Health Nutrition, 18(16), 3042-3050. https://doi.org/10.1017/S1368980014003309

Luo, R., Shi, Y., Zhou, H., Yue, A., Zhang, L., Sylvia, S., \& Rozelle, S. (2015). Micronutrient deficiencies and developmental delays among infants: Evidence from a cross-sectional survey in rural China. BMJ Open, 5(10), e008400. https://doi.org/10.1136/bmjopen-2015-008400

Lukwa, A. T., Siya, A., Zablon, K. N., Azam, J. M., \& Alaba, O. A. (2020). Socioeconomic inequalities in food insecurity and malnutrition among under-five children: within and between-group inequalities in Zimbabwe. BMC Public Health, 20(1), 1-11. https://doi.org/10.1186/s12889-020-09295-z

Mahmood, T., Abbas, F., Kumar, R., \& Somrongthong, R. (2020). Why under five children are stunted in Pakistan? A multilevel analysis of Punjab Multiple indicator Cluster Survey (MICS-2014). BMC Public Health, 20(1), 952. https://doi.org/10.1186/s12889-020-09110-9

Mendes, K. D. S., Silveira, R. C. de C. P., \& Galvão, C. M. (2008). Revisão integrativa: Método de pesquisa para a incorporação de evidências na saúde e na enfermagem. Texto \& Contexto - Enfermagem, 17(4), 758-764. https://doi.org/10.1590/S0104-07072008000400018

Morais, D. de C., Dutra, L. V., Franceschini, S. do C. C., \& Priore, S. E. (2014). Insegurança alimentar e indicadores antropométricos, dietéticos e sociais em estudos brasileiros: Uma revisão sistemática. Ciência \& Saúde Coletiva, 19(5), 1475-1488. https://doi.org/10.1590/1413-81232014195.13012013

Nepali, S., Simkhada, P., \& Davies, I. G. (2020). Association between wasting and food insecurity among children under five years: Findings from Nepal demographic health survey 2016. BMC Public Health, 20(1), 1027. https://doi.org/10.1186/s12889-020-09146-x

Nepali, S., Simkhada, P., \& Davies, I. G. (2020). Association between wasting and food insecurity among children under five years: Findings from Nepal demographic health survey 2016. BMC Public Health, 20(1), 1027. https://doi.org/10.1186/s12889-020-09146-x

Nobre, L. N., Murta, N. M. G., Souza, M. M., Ferreira, N. C., Cardoso, L. de M., \& Hamacek, F. R. (2015). Segurança alimentar em uma comunidade rural no Alto Vale do Jequitinhonha/Mg. Segurança Alimentar e Nutricional, 16(1), 18-31. https://doi.org/10.20396/san.v16i1.1809

Nuruddin, R., \& Hadden, W. C. (2015). Are pre-school girls more likely to be under-nourished in rural Thatta, Pakistan?-a cross-sectional study. International Journal for Equity in Health, 14(1), 151. https://doi.org/10.1186/s12939-015-0287-3

Oliveira, M. A. de C., \& Pereira, I. C. (2013). Atributos essenciais da Atenção Primária e a Estratégia Saúde da Família. Revista Brasileira de Enfermagem, 66, 158-164. https://doi.org/10.1590/S0034-71672013000700020

Onis, M. de (2007). Development of a WHO growth reference for school-aged children and adolescents. Bulletin of the World Health Organization, 85(09), 660-667. https://doi.org/10.2471/BLT.07.043497

Organização das Nações Unidas(2020). ONU alerta sobre desnutrição infantil aguda sem precedentes no Iêmen. https://news.un.org/pt/story/2020/10/1730892 Ochoa, H. (2017). Evaluación del estado nutricional en menores de 5 años: Concordancia de índices antropométricos en población indígena de chiapas. Nutrición Hospitalaria, 34(4). https://doi.org/10.20960/nh.700

Ochoa-Díaz-López, H. (2015). Estado nutricio de dos generaciones de hermanos(As) < de 5 años de. Nutricion Hospitalaria, (6), 2685-2691. https://doi.org/10.3305/nh.2015.31.6.9020

Organización Mundial de la alud (2019). Centro de prensa. Reducción de la mortalidad en niñez. https://www.who.int/es/news-room/factsheets/detail/children-reducing-mortality

Prost, A., Nair, N., Copas, A., Pradhan, H., Saville, N., Tripathy, P., ... Sachdev, H. S. (2019). Mortality and recovery following moderate and severe acute malnutrition in children aged 6-18 months in rural Jharkhand and Odisha, eastern India: A cohort study. PLOS Medicine, 16(10), e1002934. https://doi.org/10.1371/journal.pmed.1002934

Rodríguez Wilchez, O. O., Barrera Sánchez, L. F., \& Ospina Diaz, J. M. (2019). Knowledge, attitudes and food practices in caregivers and nutritional status in infants from Ventaquemada, Boyacá, Colombia. Archivos de Medicina (Manizales), 19(1), 74-86. https://doi.org/10.30554/archmed.19.1.2866.2019

Raymond, J., Agaba, M., Mollay, C., Rose, J. W., \& Kassim, N. (2017). Analysis of nutritional adequacy of local foods for meeting dietary requirements of children aged 6-23 months in rural central Tanzania. Archives of Public Health, 75(1), 60. https://doi.org/10.1186/s13690-017-0226-4

Samiak, L., \& Emeto, T. I. (2017). Vaccination and nutritional status of children in karawari, east sepik province, papua new guinea. PLOS ONE, 12(11), e0187796. https://doi.org/10.1371/journal.pone.0187796

Sapunar, J., Aguilar-Farías, N., Navarro, J., Araneda, G., Chandia-Poblete, D., Manríquez, V., ... Cerda, A. (2018). Alta prevalencia de trastornos nutricionales por exceso, resistencia insulínica y síndrome metabólico en escolares de la comuna de Carahue, Región de la Araucanía. Revista Médica de Chile, 146(9), 978-986. https://doi.org/10.4067/s0034-98872018000900978

Sharaf, M. F., \& Rashad, A. S. (2016). Regional inequalities in child malnutrition in Egypt, Jordan, and Yemen: A Blinder-Oaxaca decomposition analysis. Health Economics Review, 6(1), 23. https://doi.org/10.1186/s13561-016-0097-3

Silveira, F. C. P., Perosa, G. B., \& Carvalhaes, M. A. de B. L. (2012). Fatores psicossociais de risco e proteção à desnutrição infantil em mães de crianças desnutridas e eutróficas: O papel da saúde mental materna. Journal of Human Growth and Development, 22(2), 217-225. https://doi.org/10.7322/jhgd.44934

Souza, M. T. de, Silva, M. D. da, \& Carvalho, R. de. (2010). Integrative review: What is it? How to do it? Einstein (São Paulo), 8(1), 102-106. https://doi.org/10.1590/s1679-45082010rw1134

Vieira, V. L., Fiore, E. G., \& Cervato-Mancuso, A. M. (2015). Insegurança alimentar em região de alta vulnerabilidade social da cidade de São Paulo. Segurança Alimentar e Nutricional, 13(2), 34-42. https://doi.org/10.20396/san.v13i2.1830 
Research, Society and Development, v. 11, n. 1, e53011125276, 2022

(CC BY 4.0) | ISSN 2525-3409 | DOI: http://dx.doi.org/10.33448/rsd-v11i1.25276

Vasconcelos, S. M. L., Torres, N. C. P., Silva, P. M. C., Santos, T. M. P. dos, Silva, J. V. L. da, Omena, C. M. B. de, \& Oliveira, A. C. M. de. (2015). Food insecurity in households of patients with hypertension and diabetes. International Journal of Cardiovascular Sciences, 28(2). https://doi.org/10.5935/23594802.20150014

United Nations International Children's Emergency Fund (2019). Situação mundial da infância. Criança, alimentação e nutrição. Caderno Brasil UNICEF, Brasília, DF. https://www.unicef.org/brazil/relatorios/situacao-mundial-da-infancia-2019-crianca-alimentacao-e-nutricao

Vieira, V. L., Souza, M. P. de, \& Cervato-Mancuso, A. M. (2010). Insegurança alimentar, vínculo mãe-filho e desnutrição infantil em área de alta vulnerabilidade social. Revista Brasileira de Saúde Materno Infantil, 10(2), 199-207. https://doi.org/10.1590/S1519-38292010000200007

World Health Organization (2018). Global Health Observatory. Under-five mortality. https://www.who.int/data/gho

World Health Organization(2006). WHO child growth standards: Length/height-for-age, weight-for-age, weight-for-length, weight-for-height and body mass index-for-age. Methods and development. WHO (nonserial publication). Geneva, Switzerland: WHO. https://www.who.int/publications/i/item/924154693X 